\title{
Metrical interpretation of field theories
}

\section{Siparov S.V.}

State University of Civil Aviation, St-Petersburg, Russia;

E-mail: Siparov<sergey@siparov.ru>;

Traditional concept of force fields is based on Newton's mechanics and defines the dynamics of various physical systems. It is shown that it is equivalent to the use of the corresponding metric of an anisotropic space. This is a base of the geometric approach describing the motions of a physical system. Such approach makes it possible to get rid of several known paradoxes; it could be also used for the further development of the theory. Examples from classical mechanics, hydrodynamics, electrodynamics, quantum mechanics and gravitation theory are given.

Keywords: field theories, metrical interpretation, Newton's mechanics.

DOI: $10.18698 / 2309-7604-2015-1-483-501$

In classical mechanics, the following assumptions are used:

- Any environment of the body, or the observer and his instruments for non-contact measurement and observation has no effect on the body - this allows you to take the basic postulate of the existence of inertial reference frames;

- Speed of propagation of information about the coordinates of the body etc. is infinite;

- The geometry of space is Euclidean, and geometric space used in the mathematical modeling of phenomena is a direct sum of the three-dimensional coordinate space and one-dimensional time;

- Coefficient of proportionality between force and acceleration is the inertial mass of the body.

As we know, these assumptions contain arbitraries and contradictions:

- In micro-world, an observer unavoidably affects the object, and in mega-world the object unavoidably affects the observer in a non-deterministic way in both cases; therefore, the postulate of existence of inertial systems in the real physical world (1st law of motion) is not true or requires restrictions;

- Infinite speed of the signal propagation leads to logical paradoxes [1] that make it impossible to have the causality structure in the theory;

- Selection of the modeling space geometry and its dimension is arbitrary and is determined by the observer; 
- The nature of the property of inertia, i.e. whether it belongs to the body or to the outside world, can not be regarded as established, and is a philosophical assumption [2].

In modern science these problems are partly overcome, although not always in a completely satisfactory manner. For example, micro-world is successfully described using quantum mechanics, which, however, contains such paradoxes as wave-particle duality, wave function reduction, etc. In mega-world, the successes of general relativity are undeniable, but lately the interpreting of observations confronted with a problem of dark concepts (dark matter and dark energy), while the share of them had suddenly become $96 \%$ of the material content of the Universe; the carriers of them cannot be found. If we assume that the 1st law of motion dealing with the existence of inertial systems is valid approximately, i.e. starting from some distance between objects, the theory should include a constant with the dimension of distance.

Denying the infinite speed of signal propagation, first, makes it necessary to introduce a universal constant having the dimension of speed in all the theories. And secondly, it pushes to the choice of non-Euclidean geometry of space modeling the physical world in those situations where it is justified. In the simplest case, the SRT uses the 4-dimensional Minkowski geometry, while the GRT uses the 4-dimensional Riemannian geometry. Usage of these geometries allowed to strictly describing the observed effects, either no worse than in the corresponding physical theories (compare SRT and Lorentz theory of the electron) or even better (compare GRT and Newton's theory of gravitation). This indicated a new area of application of geometric ideas to the physical world.

As to the problem of inertia, the equivalence principle is used in the theories of macro- and mega-world, so the factor that makes sense of body's mass, sometimes falls out of the equations, which deprives the concept of force of the conceptual significance. In addition, there is a direct micro-world experiment (the so-called Aharonov-Bohm effect, reported in [3]), in which the motion of a particle is determined not by the forces but by potentials.

Thus, the axiomatic basis of classical mechanics (Newtonian dynamics), which allows to compare theoretical results with observations, contains two separate parts, which are not related to each other: the geometry as a way to adequately describe the real space and the processes in it and the force as the cause of the acceleration of bodies.

\section{Geometrical form of the laws of dynamics}

Let us formulate [4] the following propositions: 
Proposition 1: The geometry of space, modeling physical reality, is chosen so that the observed free body moves along the geodesic.

Example 1: In classical mechanics, when there are no forces at a distance (like gravitation), when the information spreads infinitely fast, and when the uniform and rectilinear motion of a free body can be presumably observed, the geodesic is given by $\frac{d \vec{r}}{d t}=\vec{v}_{0}$. Then, the modeling space can be chosen as a direct sum of a plane isotropic 3-dimensional space with Euclidean geometry and one-dimensional time.

Example 2: If the speed of action propagation is finite, while the free body has an observed acceleration, it is possible to endow the modeling space with due curvature and consider it to be a 4-dimensional space-time with Riemannian geometry, (as is done in GRT). Then the geodesic equation, which coincides with the equation of motion, has the form $\frac{d y^{i}}{d s}+\Gamma^{i}{ }_{k j} y^{k} y^{j}=0$, and it can be used to determine the metric tensor with the help of the observational data.

Example 3: if the observed accelerated motion of a free body shows an additional dependence on a vector field, then, alongside with curvature, the modeling space should be endowed with anisotropy. In this case, the tangent bundle appears and the geodesic equation becomes more complicated (see below).

Proposition 2: The force acting on a body is equal to the product of the matter amount measure (mass) by the acceleration determined by the equation of the geodesic.

Proposition 2 shows that now the force is only auxiliary concept in contrast to the classical 2nd law of Newtonian dynamics.

Proposition 3: Acceleration of the first body, measured with respect to the second one, is equal in magnitude and opposite in direction to the acceleration of the second body measured with respect to the first one.

Proposition 3 corresponds to the 3rd Newton's law, which refers to the equality of forces of action and reaction.

\section{Equation of motion in an anisotropic space}

The formal mathematical construction was given in $[4,5,6]$, and we would only mention that here we consider slightly curved and weakly anisotropic space with metric $g_{i j}=\eta_{i j}+\varepsilon_{i j}(x, y)$, where $\eta_{i j}=\operatorname{diag}\{1,-1,-1,-1\}$ is Minkowski metric on the main manifold, and $y^{i}=\frac{\partial x^{i}}{\partial t}$. Assuming that $\varepsilon_{i j}(x, y)=\sigma \zeta_{i j}(x, y) ; \sigma<<1$ is small (linearly approximated) anisotropic 
deformation, only the terms proportional to $\alpha_{1} \varepsilon_{i j}, \alpha_{2} \frac{\partial \varepsilon_{i j}}{\partial x^{k}}, \alpha_{3} \frac{\partial \varepsilon_{i j}}{\partial y^{k}}$ and $\alpha_{4} \frac{\partial^{2} \varepsilon_{i j}}{\partial x^{l} \partial y^{k}}$ $\alpha_{k}=O(1), \sigma \alpha_{k}<<1, k=0 \div 3$ ) will be retained in calculations.

Then in accordance with Proposition 1, the equation of motion is a geodesic of the space chosen for the modeling of physical reality. With the adopted linear approximation, the equation of geodesic takes the form

$$
\frac{d y^{i}}{d s}+\left(\Gamma_{l k}^{i}+\frac{1}{2} \eta^{i t} \frac{\partial^{2} \varepsilon_{k l}}{\partial x^{j} \partial y^{t}} y^{j}\right) y^{k} y^{l}=0
$$

where $\Gamma_{j k}^{i}=\frac{1}{2} \eta^{i h}\left(\frac{\partial \varepsilon_{h j}}{\partial x^{k}}+\frac{\partial \varepsilon_{h k}}{\partial x^{j}}-\frac{\partial \varepsilon_{j k}}{\partial x^{h}}\right)$ are the connection coefficients; here they are conventional Christoffel symbols that depend also on $y$. The list of assumptions reads:

1) Only linear terms proportional to $\alpha_{1} \varepsilon_{i j}, \alpha_{2} \frac{\partial \varepsilon_{i j}}{\partial x^{k}}, \alpha_{3} \frac{\partial \varepsilon_{i j}}{\partial y^{k}}$ and $\alpha_{4} \frac{\partial^{2} \varepsilon_{i j}}{\partial x^{l} \partial y^{k}}$ wherein $\alpha_{n}=O(1) ; i, j, l, k=0 \div 3 ; n=1 \div 4$ are retained in the metric;

2) Components $y^{1}, y^{2}, y^{3}$ can be neglected in comparison with $y^{0}$;

3) Time derivative in the equation of a geodesic can be neglected in comparison with the derivatives with respect to coordinates;

4) In the $y$-subspace $y^{0}$ derivative can be neglected in comparison with the derivatives with respect to $y^{1}, y^{2}$ and $y^{3}$.

As a result, equation (2.1) takes the form [6,7]

$$
\frac{d y^{i}}{d s}+\Gamma_{00}^{i}+\frac{1}{2} \eta^{i k} \frac{\partial^{2} \varepsilon_{00}}{\partial x^{j} \partial y^{k}} y^{j}=0
$$

Thus, it turns out that the only component of the metric tensor, which remains in the equations for the case of small curvature and weak anisotropy, is $\varepsilon_{00}$. Spatial 3D-sectional view of the equation (2.2) has the form 


$$
\frac{d \vec{V}}{d t}=\frac{c^{2}}{2}\left\{-\nabla \varepsilon_{00}+\nabla\left(\vec{V}, \frac{\partial \varepsilon_{00}}{\partial \vec{V}}\right)+\left[\vec{V}, \operatorname{rot} \frac{\partial \varepsilon_{00}}{\partial \vec{V}}\right]\right\}
$$

It gives the equation of motion in terms of the metric tensor of the anisotropic space with regard to the velocity of the probe body.

If $\varepsilon_{00} \neq \varepsilon_{00}(y)$, then $\frac{d \vec{v}}{d t}=-\frac{c^{2}}{2} \nabla \varepsilon_{00}$, and this expression leads to the known geometric formalism of GRT for gravitation, as well as to the formalism of classical mechanics as a whole. If $\varepsilon_{00} \neq \varepsilon_{00}(x)$, and $\frac{c^{2}}{2} \operatorname{rot} \frac{\partial \varepsilon_{00}}{\partial \vec{v}} \equiv \vec{\Omega}$, then the trajectory of a free particle motion is a helix whose axis is directed along vector $\vec{\Omega}$. Helix is a basic form of the trajectory of a particle's free motion in this anisotropic space.

If $\varepsilon_{00}$ component of the metric tensor becomes more complex, the helix axis ceases to be a straight line. If the metric is such that this "axis" presents a closed circle of radius $R_{M}$, which is greater than or equal to twice the radius $R$ of the helix, then the free motion takes place on the surface of a torus. If

$$
n_{1} R_{M}=n_{2} R
$$

wherein $n_{1}$ and $n_{2}$ are integers, the trajectory on the torus surface becomes closed, and since in this case $n_{1} 2 \pi R_{M}=n_{2} b, \tan (\vec{v}, \vec{\Omega})$ is a rational number. The typical linear dimension of the body, whose surface is a location of a particle free path, is equal to or larger than $2 R$.

\section{The field equations}

In classical physics, they talk about the material carriers of the cause acting on a moving body and associate them with the appropriate (force, physical) fields that can be measured. However, the direct measurement of the forces can only be performed in certain (static or stationary) cases, and usually the forces are calculated using the law of dynamics.

In the anisotropic space, every point is equipped with a vector. Let us note some mathematical circumstances related to the presence of an arbitrary vector field. Consider an 
arbitrary covariant vector with components $\vec{B}_{k}=\left(\vec{B}_{0}, \vec{B}\right), \vec{B}=\left(\vec{B}_{1}, \vec{B}_{2}, \vec{B}_{3}\right)$ and construct an antisymmetric covariant tensor, $F_{i k}=B_{k, i}-B_{i, k}$, where $B_{k, i}=\frac{\partial B_{k}}{\partial x^{i}}$. Then the expression

$$
\frac{\partial F_{i j}}{\partial x^{k}}+\frac{\partial F_{j k}}{\partial x^{i}}+\frac{\partial F_{k i}}{\partial X^{j}}=0
$$

is a geometric identity, sometimes called the Maxwell identity, valid for any geometry.

Writing this identity in components $[6,8]$, one can introduce a formal notation for a pair of new vectors constructed out of the components of tensor $F_{i k}$, namely, $\vec{F}^{(*)}=\left(F_{12}, F_{31}, F_{10}\right) ; \vec{F}^{(* *)}=\left(-F_{30}, F_{20},-F_{23}\right)$. Then we get a couple of homogeneous equations

$$
\begin{aligned}
& \frac{\partial \vec{F}^{(* *)}}{\partial t}+\operatorname{rot} \vec{F}^{(*)}=0 \\
& \operatorname{div} \vec{F}^{(* *)}=0
\end{aligned}
$$

where $t \equiv x^{0}$. The type of geometry of the space has no effect on these equations.

Let us arbitrarily choose and fix the geometry of space: let it correspond to the metric tensor $g^{i k}$, which makes it possible to switch from the covariant components of the tensor $F_{i k}$ to its contravariant components $F^{i k}$ by the formula, $F^{i j}=g^{i k} g^{j m} F_{m k}$. We introduce new additional symbols, namely, a contravariant 4-vector, $I^{i}=\left(I^{0}, \vec{j}\right), \vec{j}=\left(I^{1}, I^{2}, I^{3}\right)$, such that $I^{i}=\frac{\partial F^{i j}}{\partial x^{j}}$. Using the notation $I^{0} \equiv \rho ; I^{1} \equiv j_{x} ; I^{2} \equiv j_{y} ; I^{3} \equiv j_{z}$, get the second pair of - now inhomogeneous equations

$$
\begin{aligned}
& \operatorname{rot} \vec{F}^{(*)}-\frac{\partial \vec{F}^{(*)}}{\partial t}=\vec{j} \\
& \operatorname{div} \vec{F}^{(*)}=\rho
\end{aligned}
$$


Never going beyond the mathematical formalism, we call $\rho$ the density of sources distribution, and call $\vec{j}$ the current density. In order to describe these quantities present in equations $(3.3,3.5)$, we can formally apply the appropriate integral theorems. This will lead to the expression known as the continuity equation

$$
\operatorname{divj}+\frac{\partial \rho}{\partial t}=0
$$

The relations $(3.2,3.3,3.4)$ are always true, because they are the consequences of the mathematical identity (3.1).

If vectors $\vec{F}^{(*)}$, and $\vec{F}^{(* *)}$, composed out of the components of tensor $F_{i k}=B_{k, i}-B_{i, k}$, do not depend on $t$, then it follows from the equations $(3.2,3.3)$ that $\vec{F}^{(*)}=-\nabla \varphi$, where $\varphi$ is a certain scalar function (scalar potential) such that it satisfies the Poisson equation $\Delta \varphi=-\rho$, and, in the absence of sources $(\rho=0)$, it satisfies the Laplace equation $\Delta \varphi=0$. For a point source, $\rho=q \delta(r)$, the introduced scalar potential satisfies the "Coulomb" expression $\varphi \sim \frac{q}{r}$. The Poisson equation is linear, so, for the distributed sources, the superposition principle yields

$$
\vec{F}^{(*)}=-\nabla\left(\int \frac{\rho(r)}{\left|\vec{r}-\vec{r}_{0}\right|} d V\right)
$$

If the sources distribution is finite, homogeneous and has a spherical symmetry, then for the exterior problem we come to the "Coulomb law", mentioned above, where $q=\int_{V} \rho d V$. For the interior problem the expression (3.5) leads to $\varphi \sim q_{1} r^{2}$, where $q_{1}=\int_{V_{1}} \rho d V$, and $V_{1}$ is the volume of a sphere of radius $r$.

Vector $\vec{F}^{(* *)}$ can be represented as $\vec{F}^{(* *)}=\operatorname{rot} \vec{D}$, where $\vec{D}$ is another new vector - the socalled (3-dimensional) vector potential which satisfies the equation $\Delta \vec{D}=-\vec{j}$, and, correspondingly, 


$$
\vec{F}^{(* *)}=\operatorname{rot}\left(\int \frac{\vec{j}(r)}{\left|\vec{r}-\vec{r}_{0}\right|} d V\right) .
$$

The 4-vector $(\varphi, \vec{D}) \equiv U_{k}$ characterizes the geometric properties of the anisotropic space, each point of which is equipped with a given vector, $B_{k}=g_{i k} B^{i}$. If vector $B^{i}$ is the 4-velocity of the probe body, $y^{i}=\frac{\partial x^{i}}{\partial t}$, all the above arguments remain valid.

If there are no sources and currents, i.e. $\rho=0 ; \vec{j}=0$, then the system of equations (3.2) still has nonzero solutions that satisfy the equation

$$
g^{k i} \frac{\partial^{2} U^{i}}{\partial x^{k} \partial x^{l}}=0
$$

For the Minkowski metric, $g_{i k} \equiv \eta_{i k}$, equation (3.7) represents the wave equation written in the four-dimensional form. For other geometries, the resulting expressions may be also of interest. In particular, for the case considered in this paper, $g_{i k}=\eta_{i k}+\varepsilon_{i k}(x, y)$, and the wave equation becomes inhomogeneous. Since the correction is linear in $\varepsilon$, it must be preserved in calculations. However, if $U^{i}$ is also proportional to $\varepsilon$, then the correction can be neglected, and the equation (3.7) will be the usual wave equation.

\section{Hydrodynamics and Electrodynamics}

Let us use the notation common in hydrodynamics. We introduce $u_{i} \equiv \frac{c^{2}}{2} \frac{\partial \varepsilon_{00}}{\partial y^{i}}$, where $c$ is a fundamental parameter with the dimension of speed, and take $\Omega_{i} \equiv \frac{1}{2}$ rotu $_{i}$ and $\varphi=\frac{c^{2}}{2} \varepsilon_{00}-(\vec{v}, \vec{u})$. The relationship of these notations with symbols $\vec{B}, \vec{F}^{(*)}, \vec{F}^{(* *)}$ used above is obvious. Let us regroup equation (2.3), and multiply and divide its right hand side by a constant value, $\rho$. Then it takes the form 


$$
\frac{\partial \vec{V}}{\partial t}+(\vec{V}, \nabla) \vec{u}+2[\vec{\Omega}, \vec{V}]=-\frac{1}{\rho} \nabla\left\{\frac{\rho c^{2}}{2} \varepsilon_{00}-(\vec{V}, \vec{u}) \rho\right\}
$$

We assume vector $\vec{u}$ characterizing the anisotropy of the modeling space, be the flow rate of an incompressible fluid. We assume the value of $\rho$ be a constant density of the fluid in the neighborhood of the probe body (which is a "liquid particle"), $\rho=$ const. Then, assuming that the gradient of the two terms on the right side is the effective pressure, we obtain the Euler equation for a (rather large) region of the liquid rotating as a whole with angular velocity, $\vec{\Omega}$. The meaning of equation (3.6) is also evident.

If necessary, repeat the calculations for the components of $F_{i k}$ and get for hydrodynamics the expressions, similar to eqs. $(3.2,3.3)$, for the case when $\vec{u}$ is considered the flow rate of an incompressible fluid. Accordingly, equations (4.1) have both potential and wave solutions. Indeed, considering the speed of the liquid particle equal to the speed of its environment, $\vec{v} \cong \vec{u}$, we seek a solution of eq. (4.1) in the form

$$
\vec{V}=\vec{V} e^{i((\vec{k}, \vec{r})-\omega t)} .
$$

The amplitude $\vec{V}$ is assumed to be small enough to neglect the term, $(\vec{v}, \nabla) \vec{u} \rightarrow 0$. Then

$$
\frac{\partial \vec{V}}{\partial t}+2[\vec{\Omega}, \vec{V}]=-\nabla \phi
$$

Select a local $O z$ axis parallel to vector, $\vec{\Omega}$, and apply the rot operator to both sides of eq. (4.3), given that $\operatorname{rot}[\vec{\Omega}, \vec{v}]=\vec{\Omega} \operatorname{div} \vec{v}-(\vec{\Omega}, \nabla) \vec{v}=-(\vec{\Omega}, \nabla) \vec{v}$. Then, we obtain [9]:

$$
\frac{\partial}{\partial t} \operatorname{rot} \vec{V}=2 \Omega \frac{\partial \bar{V}}{\partial z}
$$

Dispersion equation has the form 


$$
\omega=2 \Omega \frac{k_{z}}{k}=2 \Omega \cos \theta ; \theta=\left(\vec{k}^{\wedge} \vec{\Omega}\right),
$$

and the velocity of the wave is given by

$$
\begin{aligned}
& \vec{U}=\frac{\partial \omega}{\partial \vec{k}}=\frac{2 \Omega}{k}\{\vec{v}-\vec{n}(\vec{n}, \vec{v})\} ; \vec{v}=\frac{\vec{\Omega}}{\Omega} ; \vec{n}=\frac{\vec{k}}{k} \\
& U=\frac{2 \Omega}{k} \sin \theta
\end{aligned}
$$

In such a wave, the velocity vector, $\vec{v}$ of the fluid particle retains its value and varies only in direction. In classical hydrodynamics, such waves are generated by "Coriolis force" and are called inertial waves; they have no dependence on such force characteristics as pressure. However, in the geometric theory, where both the original geodesic equation and Maxwell's identity are present, there is no Coriolis force, but there is only the metric tensor describing the anisotropic space, in which the motion of bodies takes place. In this space, the zero component of metric is the wave in accordance with the condition $\vec{v} \cong \vec{u}$, i.e. $\varepsilon_{00}=\frac{2 V^{2}}{c^{2}} e^{2 i(\vec{k}, \vec{r})-\omega t)}$.

Let us now designate, $\vec{F}^{(*)} \equiv \vec{E}, \vec{F}^{(* *)} \equiv \vec{H}$ and interpret them as the stresses of electric and magnetic fields. Then, the equations $(3.2,3.3)$ are usual Maxwell equations and the corresponding stresses are determined by formulas $(3.5,3.6)$. Using the notation $\frac{c^{2}}{2} \varepsilon_{00} \equiv \varphi$, $\frac{c^{2}}{2} \frac{\partial \varepsilon_{00}}{\partial \vec{v}} \equiv \frac{1}{c} \vec{A}$, the equation of motion of a probe body takes the familiar form

$$
\frac{d}{d t} \vec{V}=\left\{-\nabla \phi+\frac{1}{c}[\vec{V}, \operatorname{rot} \vec{A}]+\frac{1}{c}(\vec{V} \cdot \nabla) \vec{A}\right\}
$$

Here, the values of $\varphi$ and $\vec{A}$ are so-called scalar and vector potentials of the electromagnetic field. The meaning of the continuity equation (3.4) is obvious. As is known from electrodynamics (and mentioned above), in the particular case of $\varphi \neq \varphi(r) ; \operatorname{rot} \vec{A}=$ const a charged particle moves along a helix, whose axis is directed along vector, $\operatorname{rot} \vec{A}$. And electromagnetic 
waves are the classical result of theoretical physics, predicted by Maxwell on the base of the equations (3.2) and (3.3) and on the corresponding interpretation. In particular, $\vec{A}=\operatorname{Re}\left\{\vec{A}_{0} e^{i(\vec{k} \vec{r}-\omega t)}\right\}$, where $\vec{k}=\frac{\omega}{c} \vec{n}$ is the wave vector, $\vec{n}$ is a unit vector in the direction of wave propagation, $\vec{E}=i k \vec{A}$ is the stress of "electric field", $\vec{H}=i[\vec{k}, \vec{A}]$ is the stress of "magnetic field". Note that the electric field vector rotates in the plane perpendicular to the direction of wave propagation. Charge motion in the wave with circular polarization takes place in the same circumferential plane.

Thus, in the case of hydrodynamics and electrodynamics, the approach under consideration is simply another language for modeling the known observations, leading to almost the same results as the previous one. We can say that instead of the concept of a physical field now the concept of metric field is used, and the physical meaning can be assigned to it not as initially "inherent" but in view of possible interpretation.

\section{Quantum Mechanics}

The characteristic object of a micro-world, whose properties can be studied in a variety of ways, is an atom. As follows from experiments, it is a compound dynamic system (planetary model), a direct measurement of whose parameters is hardly possible, and hence, the atom can be also described in terms of metric dynamics.

For this purpose, we use the geodesic equation (2.3) and the condition of closed trajectory, eq. (2.4). Closed orbits are stable. For simplicity, we assume a trajectory to be a circle of radius $R$, and the number of revolutions $n_{1}=1$. Then we obtain:

$$
\begin{gathered}
\frac{d \vec{V}}{d t}=-\frac{c^{2}}{2} \nabla \varepsilon_{00}+\nabla\left(\vec{V}, \frac{c^{2}}{2} \frac{\partial \varepsilon_{00}}{\partial \vec{V}}\right)+[\vec{V}, \vec{\Omega}] \\
2 \pi R=n b
\end{gathered}
$$

here $b$ is a pitch of the helical trajectory, $\vec{\Omega} \equiv \frac{c^{2}}{2} \operatorname{rot} \frac{\partial \varepsilon_{00}}{\partial \vec{v}}, n$ is a positive integer. Thus, the basis of quantization is equation (5.2), which has the purely mathematical nature.

Solving the equation (5.1), we search for $\vec{v}$ in the form

$$
\vec{V}=\vec{V} \exp i\{(\vec{k}, \vec{r})-\omega t\},
$$


and we search for a correction $\varepsilon_{00}$ to the component of the metric tensor in the form

$$
\varepsilon_{00}=\frac{1}{2} \frac{V^{2}}{c^{2}}=\frac{1}{2} \frac{V^{2}}{c^{2}} \exp 2 i\{(\vec{k}, \vec{r})-\omega t\}
$$

considering the real parts in both cases. Then, $\nabla \varepsilon_{00}=2 i \vec{k} \varepsilon_{00}, \nabla\left(\vec{v}, \frac{c^{2}}{2} \frac{\partial \varepsilon_{00}}{\partial \vec{v}}\right)=c^{2} 2 i \vec{k} \varepsilon_{00}$, $\vec{\Omega}=\frac{i}{2}[\vec{V}, \vec{k}] \exp \{i\{(\vec{k}, \vec{r})-\omega t\}\}$, and equation (5.1) gives

$$
\frac{d \vec{V}}{d t}=i c^{2}\{\vec{W}+[\vec{W},[\vec{W}, \vec{k}]]\} \varepsilon_{00}
$$

where $\vec{w}=\frac{\vec{V}}{V}$, and the dispersion relation has the form

$$
\omega \vec{W}=-\frac{1}{2} V\{\vec{k}+[\vec{W},[\vec{W}, \vec{k}]]\} \exp i\{(\vec{k}, \vec{r})-\omega t\}
$$

The obvious connection between the characteristics of the wave and the helix, gives $b=\frac{2 \pi}{k}$, so, we find an estimate for the size of an atom from equation (5.2):

$$
R=\frac{n}{k}
$$

The estimates, that follow from eqs. $(5.3,5.4)$ in this case, are

$$
\begin{gathered}
V=V \cos n \\
\varepsilon_{00}=\frac{1}{2} \frac{V^{2}}{c^{2}} \cos ^{2} n .
\end{gathered}
$$


Let us, as usual, assume that the atomic transition from one state to another state, characterized by different number $n$, changes the atom's energy. Now the energy is

$$
\begin{gathered}
T=\frac{m V^{2}}{2}\left\{\sin ^{2}(\vec{V}, \vec{\Omega})+\cos ^{2}(\vec{V}, \vec{\Omega})\right\}=\frac{m V^{2}}{2} \cos ^{2} n \\
U=\frac{m c^{2}}{2}\left\{\varepsilon_{00}-\left(\vec{V}, \frac{\partial \varepsilon_{00}}{\partial \vec{V}}\right)-\phi_{(a)}\right\}=\frac{m c^{2}}{2}\left(\varepsilon_{00}-2 \varepsilon_{00}+\varepsilon_{00}\right)=0
\end{gathered}
$$

It turns out that in this case the motions along the various closed paths are determined by the same (zero) value of the "potential energy". This circumstance arising in metric dynamics is a mathematical reflection of the "physical" Bohr postulate of the existence of stationary orbits, i.e., of the stability of such a dynamic system as an atom, and even in several possible states. Thus,

$$
\left|E_{n_{1}}-E_{n_{2}}\right|=\frac{m V^{2}}{2}\left|\cos ^{2} n_{1}-\cos ^{2} n_{2}\right|
$$

If the further interpretation suggests that the transition from one state to another results in the emission of the energy difference from the system in the form of a quantum, then (5.12) shows that the spectrum of radiation should be almost continuous. Indeed, the difference of the squares of cosines of integers can be made almost any (less than unity) with an appropriate choice of integers. This is consistent with the observable fact that, e.g. xenon, and other light sources based on inert gases provide a substantially continuous spectrum of radiation.

Since Planck's quantum hypothesis is natural for metric dynamics, and the photon energy is $\mathrm{E}=h v$, let us use the value of $h$, obtained from experiments with radiation, to estimate the rate of finite motion of the particle with regard to $\mathrm{E}=\left|E_{n_{1}}-E_{n_{2}}\right|$. The frequency of visible light, $v \sim 10^{15} \mathrm{~s}^{-1}$, and electron mass is equal to $m_{e} \sim 10^{-30} \mathrm{~kg}$, both measured independently, hence, we obtain $V \sim 10^{6} \mathrm{~m} / \mathrm{s}$. Then with the help of $(5.6,5.7)$, we get an estimate $R \sim \frac{1}{k} \sim \frac{V}{v} \sim 10^{-10} \mathrm{~m}$ for the size of an atom, which coincides with the known one.

The difference between the results of the theory of hydrogen atom proposed by Bohr and based on the analysis of its spectra, and the results obtained here, stems from the fact that a hydrogen atom is described in the framework of the two-body problem with Coulomb interaction 
potential. However, for more complex situation, the problem of many bodies (with the Coulomb potential) has no analytical solution. So, the use of the classical approach to describe transitions in atoms of inert gases ${ }^{2}$, occurring within the "electron shell" containing several bodies, is impossible. This was one of the reasons why the further development of quantum mechanics has followed an abstract and even contradictory way rejecting visual representations.

In metrical dynamics with the geodesics $(2.1,2.2)$ as the equations of motion, the trajectory of the free motion is a helix of general form. Therefore, the motion of a particle is naturally characterized by a concept of phase (compare to [10]), and plane waves correspond to the projections of the trajectory of motion on various planes ${ }^{3}$. At the same time, the wave properties of the moving micro-particle such as rounding the obstacles and the emergence of a variety of diffraction and interference patterns, depending on the boundary conditions, can be now described by selecting the suitable geometry of modeling space. Notice the wave-like form of the correction to the metric.

\section{Gravitation}

The approach based on the geometry discussed here was proposed and used as the theory of gravitation, and it was natural to call it anisotropic geometrodynamics (AGD). It is described in more detail in papers $[7,11]$ and in the monograph [6]. Here we shall mention only the main points and present the results previously obtained in these studies.

The equation of motion is again determined by the equation of the geodesic eq. (2.3), and the gravitational force in accordance with Proposition 2 has the form

$$
\vec{F}_{g}=\frac{m c^{2}}{2}\left\{-\nabla \varepsilon_{00}+\nabla\left(\vec{V}, \frac{\partial \varepsilon_{00}}{\partial \vec{V}}\right)+\left[\vec{V}, \operatorname{rot} \frac{\partial \varepsilon_{00}}{\partial \vec{V}}\right]\right\}
$$

It contains the contributions of three terms. If there are no additional assumptions, neither one of the terms can be neglected in comparison with the others. The physical meaning, which can be attributed to the additional terms in the interpretation, is also clear. Namely, the equivalence principle must be generalized: since the force of inertia may depend on the velocity of the body, the force of gravity should also depend on it. For this reason, AGD may also be called a generalized

\footnotetext{
${ }^{2}$ And in other complex atoms

${ }^{3}$ The formalism of the regular quantum mechanics corresponds to the description of these very projections.
} 
theory of equivalence (GTE). Depending on the angle between the velocity vector of a particle, and vectors associated with $\frac{\partial \varepsilon_{00}}{\partial \vec{v}}$, their contributions, comparable in magnitude, can have different signs. This reflects the possibility to observe not only attractive action but also repulsive or tangential action. Using the concepts of "metric field" given in Section 3, one can calculate any model situation, in which the distributions of moving sources $\rho$ and $\vec{j}$ allow to calculate $\vec{F}^{(*)}$ and $\vec{F}^{(* *)}$. For a simple system where the test body moves along a closed circular current surrounding a singular source, the equation of motion will lead to $v^{2}=\frac{C_{1}}{r} \pm v C_{2}$, where $C_{1}$ and $C_{2}$ are constants, and the sign depends on the direction of motion of a probe body. Solutions have the form

$$
\begin{gathered}
V=\frac{C_{2}}{2}\left(1-\sqrt{1 \pm \frac{4 C_{1}}{r C_{2}^{2}}}\right) \\
V=\frac{C_{2}}{2}\left(1+\sqrt{1 \pm \frac{4 C_{1}}{r C_{2}^{2}}}\right)
\end{gathered}
$$

If we apply this model to describe the motion of stars in a spiral galaxy, then eq. (6.3) corresponds to the Newtonian result and means that the velocity of the orbital motion decreases with increasing distance from the center. This is consistent with general relativity and Newton gravitation. At the same time, formula eq. (6.4) describes the situation when the speed of the orbital motion of a star tends to a constant, which corresponds to the observed flat rotation curves. In [6,7] and in [11] it is shown that there is also a quantitative agreement with observations. Writing down the explicit expressions for the constants $C_{1}$ and $C_{2}$ through the parameters of the problem and assuming that the luminosity of a spiral galaxy is proportional to its area, we get the Tully-Fisher law, $v_{\text {orb }} \sim L_{\text {lum }}{ }^{1 / 4}$, known from observations, which has no explanation in general relativity.

Thus, the use of anisotropic geometry to describe the astronomical observations on the galactic scale makes it possible to adequately describe them, and no "dark matter" is required. Other results obtained in the framework of the AGD in [6,7] and [11] are summarized in the following table: 
Table 1.

\begin{tabular}{|c|c|c|}
\hline AGD Results & Observations & Modern interpretation \\
\hline $\begin{array}{l}\text { 1. If the gravitational field does not } \\
\text { depend on the velocities of the bodies, } \\
\text { the AGD equations become the GRT } \\
\text { equations. }\end{array}$ & $\begin{array}{l}\text { Confirm the theory on the scale of } \\
\text { the solar system }\end{array}$ & GRT \\
\hline $\begin{array}{l}\text { 2. When a body performs the } \\
\text { gravitational acceleration maneuver } \\
\text { driving in the planetary system, an } \\
\text { additional acceleration directed } \\
\text { toward the center, which is } \\
\text { proportional to } c H \text { should be } \\
\text { observed. ( } H \text { is Hubble constant) }\end{array}$ & $\begin{array}{l}\text { Effect of "Pioneers" which has just } \\
\text { the order of } c H \text {. }\end{array}$ & $\begin{array}{l}14 \text { various explanations that take } \\
\text { into account the following aspects: } \\
\text { technical, data processing, space } \\
\text { objects, and «new physics". They } \\
\text { are comparable in order of } \\
\text { magnitude, which does not allow } \\
\text { choosing only one of them. }\end{array}$ \\
\hline $\begin{array}{l}\text { 3. The rotation curves for spiral } \\
\text { galaxies are flat }\end{array}$ & Yes. & $\begin{array}{l}\text { 1. There is dark matter, } \\
\text { whose mass is } 4 \text { to } 7 \\
\text { times the mass of the } \\
\text { luminous (baryonic) } \\
\text { matter in the galaxy. Dark } \\
\text { matter particles possess } \\
\text { exotic properties and have } \\
\text { not yet been found in a } \\
\text { direct experiment } \\
\text { 2. In the MOND theory the } \\
\text { change of the Newtonian } \\
\text { dynamics equations is } \\
\text { proposed by introducing } \\
\text { an additional term, } \\
\text { ensuring fit to the } \\
\text { observations. }\end{array}$ \\
\hline $\begin{array}{l}\text { 4. The orbital velocities of stars and } \\
\text { gas, corresponding to the flat rotation } \\
\text { curves of spiral galaxies must comply } \\
\text { with centripetal acceleration of order } \\
\mathrm{cH} \text { at distances of the order of the } \\
\text { radius of the galaxy. }\end{array}$ & Yes. & Interpretation is missing. \\
\hline $\begin{array}{l}\text { 5. If the luminosity of a galaxy is } \\
\text { proportional to its mass, and the mass } \\
\text { of a spiral galaxy is distributed in the } \\
\text { plane, the luminosity should be }\end{array}$ & $\begin{array}{l}\text { Tully-Fisher law resulting from } \\
\text { the observations. }\end{array}$ & $\begin{array}{l}\text { Interpretation is missing. } \\
\text { Note that in these observations, the } \\
\text { hypothetical dark matter present in }\end{array}$ \\
\hline
\end{tabular}




\begin{tabular}{|c|c|c|}
\hline $\begin{array}{l}\text { proportional to the fourth power of } \\
\text { the orbital velocity of the stars on the } \\
\text { periphery. }\end{array}$ & & $\begin{array}{l}\text { a galaxy does not manifest itself, } \\
\text { which contradicts its supposed } \\
\text { property to have a gravitational } \\
\text { action. }\end{array}$ \\
\hline $\begin{array}{l}\text { 6. The galaxies with large angular } \\
\text { momentum should have arms. }\end{array}$ & Yes, spiral galaxies. & $\begin{array}{l}\text { The theory of density waves. } \\
\text { It does not predict the bars. }\end{array}$ \\
\hline 7. Spiral galaxies should have bars. & Yes. & Interpretation is missing. \\
\hline $\begin{array}{l}\text { 8. The motion of individual objects in } \\
\text { the plane of the spiral galaxy must } \\
\text { contradict the Kepler law }\end{array}$ & $\begin{array}{l}\text { Observations of globular clusters } \\
\text { in the galactic plane disclose a } \\
\text { violation of the Kepler law } \\
\text { statistics: the number of clusters in } \\
\text { the center of the galaxy is } \\
\text { significantly greater than in the } \\
\text { periphery. }\end{array}$ & Interpretation is missing. \\
\hline $\begin{array}{l}\text { 9. The motion of objects in the plane } \\
\text { of a spiral galaxy and in the plane } \\
\text { perpendicular to it should be different }\end{array}$ & $\begin{array}{l}\text { Observations of globular clusters } \\
\text { in the perpendicular plane } \\
\text { correspond to Kepler's law as } \\
\text { opposed to i. } 8\end{array}$ & Interpretation is missing. \\
\hline $\begin{array}{l}\text { 10. In some gravitational lenses } \\
\text { having the necessary orientation, } \\
\text { there must be a significant excess of } \\
\text { refraction compared with the estimate } \\
\text { following from GRT }\end{array}$ & Yes. & $\begin{array}{l}\text { Considered to be related to the } \\
\text { action of dark matter and is used to } \\
\text { estimate its amount. }\end{array}$ \\
\hline $\begin{array}{l}\text { 11. Gravitational lenses, which are } \\
\text { spiral galaxies, with the profile } \\
\text { orientation should give the asymmetry } \\
\text { in the image. }\end{array}$ & $\begin{array}{l}\text { Yes. For example, the Einstein } \\
\text { Cross. }\end{array}$ & Interpretation is missing. \\
\hline $\begin{array}{l}\text { 12. In view of mass and energy } \\
\text { equivalence, the clusters of galaxies } \\
\text { should have larger mass than it can be } \\
\text { assessed by their luminosity, and } \\
\text { larger than a correction, which } \\
\text { follows from the GRT. }\end{array}$ & F. Zwicky oobservations. & $\begin{array}{l}\text { "Hidden mass" (in accord with } \\
\text { F.Zwicky) and dark matter. }\end{array}$ \\
\hline $\begin{array}{l}\text { 13. In collisions of individual } \\
\text { galaxies, there should be manifested } \\
\text { the excess of "mass-energy" } \\
\text { associated with mutual movement }\end{array}$ & $\begin{array}{l}\text { Observation of the collision of } \\
\text { galaxies in the Bullet cluster made } \\
\text { by "Chandra" observatory. }\end{array}$ & Dark matter. \\
\hline $\begin{array}{l}\text { 14. The red shift in the emission of } \\
\text { distant objects should increase }\end{array}$ & $\begin{array}{l}\text { Empirical law discovered by } \\
\text { E.Hubble }\end{array}$ & $\begin{array}{l}\text { Cosmological expansion of the } \\
\text { Universe }\end{array}$ \\
\hline
\end{tabular}




\begin{tabular}{|l|l|l|}
\hline $\begin{array}{l}\text { linearly with distance, which is } \\
\text { associated with vortex motion of } \\
\text { object on the scale of galaxies and } \\
\text { higher. }\end{array}$ & $\begin{array}{l}\text { Deviations from the linear Hubble } \\
\text { law, relevant to this hypothesis } \\
\text { gravitational lens, resulting in } \\
\begin{array}{l}\text { incorrect (overvalued) determination } \\
\text { of the distance to the corresponding } \\
\text { light sources. }\end{array}\end{array}$ & $\begin{array}{l}\text { Expansion of the Universe is } \\
\text { accelerated due to the dark energy } \\
\text { (of repulsion). }\end{array}$ \\
\hline $\begin{array}{l}\text { 16. The distribution of matter near the } \\
\text { nuclei of spiral galaxies can have a } \\
\text { characteristic form (the "infinity" } \\
\text { sign) }\end{array}$ & $\begin{array}{l}\text { Discovered by } \\
\text { observatory under the supervision } \\
\text { of cold gas clouds in the center of } \\
\text { our galaxy. }\end{array}$ & \\
\hline
\end{tabular}

From a formal point of view, the use of force fields is equivalent to the use of appropriate space geometry selected for the simulation of physical reality. In a sense, it returns to the old philosophical debate about the materiality of the field and about the action at a distance. As before, it may be subject to personal preferences of the researcher. But just as before, the involvement of new mathematical apparatus may enable a new theoretical progress and compare the results with the results of appropriate experiments.

\section{References}

1. Silagadze Z. (2008). Relativity without tears. Acta Phys.Pol. 39, 812.

2. Mach E. (1975). Mechanics. In the coll. "Albert Einstein's theory of gravity." Moscow: Mir [World].

3. Chambers R.G. (1960). Shift of an Electron Interference Pattern by Enclosed Magnetic Flux. Phys. Rev. Lett., 5, 3.

4. Siparov S.V. (2013). On the foundations of the generalized theory of equivalence (anisotropic geometrodynamics). HCNGP, 19, 162-183.

5. Siparov S., Brinzei N. (2008). arXiv, [gr-qc]: 0806.3066v1.

6. Siparov S. (2011). Introduction to the Anisotropic Geometrodynamics. London-New JerseySingapore: World Scientific.

7. Siparov S.V. (2008). On the Anisotropic Geometrodynamics. HCNGP, 10, arxiv [gr-qc]: $0809.1817 \mathrm{v} 3$. 
8. Einstein A. (1979). The general theory of relativity. In the coll. "Albert Einstein's theory of gravity." Moscow: MIR [World].

9. Landau L.D., Lifshitz E.M. (1986). Gidrodinamika [Hydrodynamics]. Moscow: Nauka [Science].

10. De Broglie L. (1986). Heisenberg uncertainty relations and probabilistic interpretation of quantum mechanics. Moscow: MIR [World].

11. Siparov S.V. (2009). The law of gravitation and the model of source in the anisotropic geometrodynamics. HCNGP, 12, 140-160. 